\title{
Social Networks and KMS Use in US IT Services
}

\author{
William J. Dixon
}

Americas IT, Ernst \& Young LLP, 1401 McKinney Street, Suite 1200, Houston, Texas, 77010, US, bill.dixon@ey.com

\begin{abstract}
Little is known about how people, contexts, and tools impact decisions to use a Knowledge Management System (KMS). The purpose of this study was to better understand information retrieval when solving difficult problems. Key research questions focused on social structure, interpersonal relationships, and nature of the KMS. In this sequential exploratory study, semi-structured interviews were conducted and surveys were distributed to a purposive sample of 299 technology support personnel in a large accounting firm. Thematic analysis was applied against interview outcomes, and survey responses were analyzed using ANOVA and confirmed with the Kruskal-Wallis test. Social structure analysis showed fewer structural holes within networks among routine KMS users. Contrary to social resource theory, information was rarely sought from supervisors. Reciprocal information exchange accompanied asking for help, but not when information was retrieved from the KMS. In addition, formal designation of experts, electronic instant messaging (IM), and KMS minimized the impact of geographic disparity. The KMS facilitated the distribution of information and enabled learning but was not uniformly adopted. Recommendations for practice include the strategic designation of experts and refinement of mechanisms available for information retrieval.
\end{abstract}

\section{Introduction}

When a problem in the workplace is encountered, service providers make choices. Knowledge on which to base such decisions is imperfectly shared over time, and across people and organizations [10]. Employees often seek advice by asking their colleagues [14]. However, too much reliance on colleagues for advice may not result in an optimum outcome. People trying to solve a problem may not know who has the most pertinent expertise to help, resulting in an unconscious decision to settle for a less than optimum solution. The Knowledge Management (KM) literature abounds with descriptions of systems designed to guide decision makers [2, 
15]. Most often, these systems include databases from which it is intended that decision makers will receive value. Value can be realized through shortened time to troubleshoot or better solutions to reoccurring problems. Designers of KM tools assume such systems will be used as they were intended. However, tools are often applied in practice differently than organizational leadership anticipated [2,6]. This gap between organizationally-supported practice and actual behavior has been attributed to the "partial articulation of the espoused rules that govern behavior" [16, p. 601]. This research project examined the rules that govern the behavior of potential KMS users.

Borgatti and Cross supported the idea that "relationships are important for the acquisition of information and that the creation of knowledge is a social process" [5, p. 440]. Nevertheless, they concluded that little was known about the structural or interpersonal relationships that facilitated information exchange. Others have concluded "knowledge seeking and use, the employment of tools to support KM, and the outcomes of KM have yet to be carefully examined" [1, p. 197]. The problem examined in this study was this uncertainty around decisions to use a KMS. An extended literature review on these topics is available at http://home.comcast.net/ $\sim$ wm_dixon/. The following research questions framed this research:

1. How were the physical characteristics of the social networks of those who chose to use a KMS different than those who did not use a KMS?

2. How were the relational characteristics of the social networks of those who chose to use a KMS different than those who did not use a KMS?

3. What was the relationship between KMS utilization and the perceived accessibility of colleagues?

4. What was the relationship between KMS utilization and the perceived costs of asking colleagues for help?

5. What were the perceived benefits of KMS use?

\section{Research Method}

An exploratory, semi-structured interview strategy was used to first investigate how social context impacts decisions to use a KMS. Observations drawn from the interviews were then further examined and explored by conducting a census survey.

The behaviors of people associated with support services in the IT organization of a large accounting firm were examined in this study. The internal support organization maintained the IT infrastructure used by over 25,000 staff in the United States. Approximately 400 support technicians, spread between 72 offices, supported the day-to-day IT operations within the firm. All support technicians used the same call tracking and KMS to document their support activities. Technicians 
had the opportunity to both read support articles stored in the KMS and to recommend articles for inclusion. The article proposal process triggered a formal review of proposed support articles by specialists in each domain. Specialists then leveraged content managers and technical writers to publish a support article. Technicians were geographically dispersed throughout the continental U.S. and Hawaii. The sampling frame consisted of lists of login IDs from the call tracking system. A probability sample was drawn from this sampling frame. Stratification was employed, based on the concentration of support personnel in each office location. Three strata, each representing about $33 \%$ of the population, were established; offices in which 22 or more support personnel were based, where between 9 and 22 technicians were based, and where 8 or fewer technicians were based.

Participants of semi-structured interviews were identified using a proportionate stratified random sample. Five participants were randomly selected from each of the three strata to participate in face-to-face interviews which were conducted in the home office of the respondent. For the survey, a census sampling strategy was implemented. All members of the sampling frame were encouraged to complete an electronic self-administered questionnaire.

During the first phase of the study, semi-structured interviews were conducted to uncover attitudes toward KMS utilization and the social networks in which participants operated. The semi-structured interviews consisted of open-ended questions, and the topics were drawn from the literature. These questions focused on the quality of relationships, the nature of the KMS, and the nature of the information managed by participants. The objective of the interviews was to determine if there were particular concepts that were most important in deciding to use a KMS. Concepts in this category centered on trust, communication, relationships, job assignments, and individual predispositions. Data from the interviews was transcribed, coded, and compiled.

Subsequent to the interviewing process, a survey instrument was developed to determine the respondent's attitudes toward the outcomes of the interviews, physical structure of a respondent's social network, and relational structure of a respondent's network. The survey instrument used is available at http://home.comcast.net/ wm_dixon/. Demographic information sought included (a) employee tenure, (b) the professional rank, (c) how often the respondent used the KMS, and (d) the number of technicians based in their home office.

Descriptors of social network relationships, drawn from social network analysis (SNA), were calculated to compare the characteristics of social networks. The number of direct social linkages to each person named (agent or node) was calculated (vertex degree). Similarly, correlations between measures of network structure, relationships, and KMS use were sought. ANOVA of self-reported KMS utilization was compared between survey respondents from each of the three strata. The appropriate confirmatory tests were conducted in search of relationships between demonstrated KMS utilization and (a) vertex degree, (b) network centrality, (c) network density, (d) network cohesion, and (e) pertinent relationships that emerged from the interviews. 


\section{Results}

Interviews were conducted with 15 technologists from 11 different office locations spread throughout the continental United States. During each interview, notes were taken by the interviewer. In addition, each session was recorded and transcribed. Information seeking behaviors were dependent on the circumstances. One respondent exhibited an innate tendency to think first about a person who had the information he sought and then to attempt to retrieve that information from the KMS.

[My] first natural reaction is to think about who I think would know. That's my first natural reaction. What I really tend to do is look at the knowledge bases we have available. Depending on what the situation is. If it's a [Lotus] Notes issue, check out the Notes Knowledge Base. If it is something that is one of those general questions that I just forgot the answer, I know it's in there; I'll go into [the KMS] to find that information. (W.

Dixon, personal communication, November 14, 2006)

The tendency to look for information in a KMS when the respondent was confident the information being sought was in the database was restated by participants from all strata. Furthermore, there was awareness among all interview participants of the professional rank of their information sources. Technical information was rarely sought from a participant's direct supervisor or person of senior rank.

Textual analysis was performed on the transcripts from the interviews. Respondents described their information retrieval protocol when being pushed to complete tasks quickly differently than when deadlines or heavy workloads were absent. Examples of why respondents preferred interpersonal interaction during information retrieval included (a) speed of information retrieval, (b) the ability to convey more specifically and completely what information was sought and (c) the opportunity to ask follow-up questions. The ability to engage in discussion with a knowledgeable colleague enhanced the learning process and helped the information seeker gain clarity on the information they needed. Two respondents indicated peer interaction facilitated KMS use when they were directed to a specific document in the KMS. Another interview participant described information retrieval from colleagues as a collaborative approach to the problem resolution process: "It is like having an extra set of hands. Two minds are better than one when trying to solve a problem."

There was greater consistency in terminology used by technologists who were from small offices than the medium and large offices. Technologists from small offices discussed direct interaction with their customers more often than their counterparts from larger offices. Peer interaction was discussed far more frequently by personnel in large offices and to a lesser extent by those who worked in medium-sized offices.

When in the presence of a client, respondents behaved differently than if they were on the telephone or if the client was not physically present. Information was sought from people when respondents knew a colleague had special information. 
The KMS was often used when the respondent had sufficient information to formulate cohesive questions around the information being sought.

Individual communication styles were also criteria by which methods of information retrieval were determined. A respondent indicated interoffice conflicts had resulted from misconstrued messages among technologists. Consequently, some team members preferred face-to-face discussion over electronic instant messaging (IM) or e-mail.

Two classifications of affinity groups had been chartered by management as centers for information sharing. Members of these affinity groups were considered experts in their domains. One affinity group consisted of subject matter experts (SME teams) associated with selected products. Another was a group of people to whom problems were escalated by technologists once they had exhausted all troubleshooting resources, called the Virtual Technology Assistance Center (V-TAC). A respondent from a large office observed that many individuals who served in these expert roles were located in the same office location as he. Several kinds of IM sessions were discussed. More prevalent in small and medium-sized offices, some service managers had sanctioned IM sessions as information sharing mechanisms. One of the large offices had branded such sessions as Tech Chat and all technologists at that location where invited to participate.

Information retrieval strategies served multiple purposes. Relationships were nurtured as well as information obtained when respondents received information from colleagues. A participant mentioned she occasionally reached out to technologists outside of her area simply because she liked to meet new people. Another participant found the credibility of his peers reinforced through the information stored in the KMS.

Anchored to the research questions, four key operational themes emerged from the interview process and literature review: (a) preferences for information sources; (b) characteristics of a person as an information source; (c) information retrieval behaviors; and (d) situational nature of information retrieval.

The survey required the description of at least one interpersonal relationship. Respondents had the capability to complete a survey electronically for a two-week period. At that time, 299 technicians met the qualifications to complete the survey. Ultimately 129 surveys were completed and 17 additional respondents formally declined to participate. Those who reported weekly KMS use were labeled as routine KMS users (114). Non-routine KMS users were defined as those who selfreported having used the KMS on a quarterly basis or less, corresponding with the remaining 15 respondents.

Survey responses obtained from the routine KMS users were compared to responses from the non-routine KMS users. Both parametric and nonparametric statistical tests were used to mitigate the risks inherent in comparing unbalanced data, and assumptions regarding normal distributions, and similar variances [11].

Those who routinely used the KMS had worked for the firm for a shorter period of time than those who did not routinely use the KMS. The median number of years that routine KMS users had worked for the firm was 8.75 years, whereas the 
median tenure of those who reported they do not routinely use the KMS was 10 years. Similarly, the most recurrent response to the question regarding tenure with the firm was 7 years for KMS users and 11 years for non-routine KMS users.

\subsection{Physical Characteristics of Social Networks}

When describing their social networks, 700 unique descriptors were reported by respondents and linked to 1227 interpersonal relationships. The most recurrent person identified was named by 19 respondents. No statistical difference was found between the vertex degree of routine KMS users and respondents that did not routinely use the KMS.

Non-routine KMS users reported having known most of the members of their social networks longer than the routine KMS users. A statistical difference in how long KMS users had known the members of their networks was confirmed. The dispersion of years of employment was greatest for routine KMS users whereas the responses of non-routine KMS users were more heavily clustered at five or more years.

The frequency of interaction among network participants was most evenly distributed for routine KMS users. Non-KMS users interacted more frequently with their network members than those who routinely used the KMS. In Table 1, the characteristics of reported information networks are listed.

Table 1. General Social Network Characteristics

\begin{tabular}{|c|c|c|c|c|c|c|}
\hline$\underline{\text { Strata/KMS Use }}$ & Nodes & Edges & Outsiders & Centrality & Density & Cohesion \\
\hline Large Office/KMS User & 35 & 44 & 0 & 4.5 & 0.0370 & 0 \\
\hline Med. Office/KMS User & 158 & 236 & 4 & 9.5 & 0.0095 & 4 \\
\hline Med. Office/Non-User & 14 & 11 & 0 & 2.8 & 0.0604 & 0 \\
\hline Small Office/KMS User & 234 & 421 & 2 & 19.4 & 0.0077 & 6 \\
\hline
\end{tabular}

Edges represented the number of relationships drawn between people. Outsiders were created when a respondent volunteered the names of their contacts, but that individual was not identified by other survey respondents. Centrality reflected the extent to which information in a network was distributed [4] and was lowest among those who did not routinely use the KMS. In Table 1, centrality was measured as relative entropy. Relative entropy accommodates the comparison of centrality between networks of differing sizes. The degree of connectedness of each network was represented by density and was highest for those who did not routinely use the KMS. Cohesion reflected the extent to which directed networks were symmetrical. Symmetry was greatest in networks with many nodes. 


\subsection{Relational Characteristics}

Non-routine KMS users reported relationships with individuals in roles encompassing formalized expertise less often than did KMS users. Whereas non-routine KMS users reported relationships with V-TAC or SME team members in $37 \%$ of the relationships reported, KMS users reported that $63 \%$ of their relationships were with personnel in those formal roles. Personnel based in small offices leveraged those in formalized roles more often than their counterparts who were based in medium and large offices. Two respondents wrote comments indicating they also considered prior experience of information providers in formalized roles as an inducement to rely on those individuals for information.

Personnel who routinely used the KMS considered the legitimate roles of their contacts more important than the availability of that contact. In Table 2, the proportion of each population that reported any of these attributes of social relationships as most important is summarized. When seeking information, those who did not routinely use the KMS reported the availability of their contacts as more important than the legitimate role of that individual.

Table 2. Characteristics Reported as Most Important by KMS Use

\begin{tabular}{|c|c|c|c|c|c|}
\hline Usage & Availability & Legitimate Role & Having Met & $\begin{array}{c}\text { Face-to-Face } \\
\text { Interaction }\end{array}$ & Supervisor \\
\hline $\begin{array}{l}\text { Routine KMS } \\
\text { Users }\end{array}$ & $32 \%$ & $41 \%$ & $5 \%$ & $10 \%$ & $1 \%$ \\
\hline $\begin{array}{l}\text { Non-routine } \\
\text { KMS User }\end{array}$ & $47 \%$ & $27 \%$ & $5 \%$ & $11 \%$ & $1 \%$ \\
\hline
\end{tabular}

Survey responses demonstrated a link between office size, the availability of an information source, and their legitimate role. In Table 3, the proportion for each stratum that reported relational criteria as most important is summarized. Personnel from small offices most often rated the legitimate role of information providers more important than their availability whereas personnel from the medium strata considered availability more important.

Table 3. Most Important Characteristics by Strata

\begin{tabular}{|c|c|c|c|c|c|c|}
\hline Strata & $\mathrm{N}$ & Availability & Legitimate Role & Having Met & $\begin{array}{c}\text { Face-to-Face } \\
\text { Interaction }\end{array}$ & Supervisor \\
\hline Large & 9 & $22 \%$ & $33 \%$ & $11 \%$ & 0 & 0 \\
\hline Medium & 49 & $47 \%$ & $31 \%$ & $6 \%$ & $10 \%$ & 0 \\
\hline Small & 71 & $25 \%$ & $46 \%$ & $3 \%$ & $13 \%$ & $1 \%$ \\
\hline
\end{tabular}

Contrary to the literature, supervisors were the least preferred information source for all respondents. Few survey respondents described information seeking 
relationships with their supervisors $(10 \%$ of the relationships reported by nonroutine KMS users and $6 \%$ of the relationships reported by routine KMS users).

\subsection{Accessibility of Colleagues}

Personnel who routinely used the KMS considered the legitimate roles of their contacts more important than the availability of that contact. Conversely, when seeking information, those who did not routinely use the KMS reported the availability of their contacts as more important than the legitimate role of that individual.

Interview participant's revealed accessibility could be obtained through direct interpersonal contact or by electronically mediated mechanisms. The majority of survey respondents participated in IM sessions with personnel from other offices to obtain support information. As detailed in Table 4, there was a higher incidence of IM participation among routine KMS users than those who did not routinely use the KMS.

Table 4. Participation in Electronic Chat (IM) Sessions

\begin{tabular}{lllll}
\hline Usage & All & Large Office & Medium Office & Small Office \\
\hline Routine KMS Users & $77 \%$ & $71 \%$ & $70 \%$ & $83 \%$ \\
Non-KMS Users & $42 \%$ & $67 \%$ & $63 \%$ & $25 \%$ \\
\hline
\end{tabular}

The availability of a colleague was a precursor to asking a colleague for information for those who did not routinely use the KMS. On average, routine KMS users did not agree that colleague availability led to asking him or her for information.

\subsection{Costs of Asking for Help}

Almost all respondents indicated they went out of their way to help a peer find information if a peer had provided information in the past. Claims of such reciprocity were not limited to any strata or contingent on KMS use. Few respondents agreed that asking a peer for help made the respondent appear less competent. There was no significant difference between cohorts in either of these areas.

Speed was a recurrent theme volunteered by those who participated in the interview process. Those who did not routinely use the KMS agreed information was most quickly retrieved from a colleague. In contrast, those who routinely used the KMS were indifferent to this idea. 


\subsection{Benefits of KMS Use}

There was general agreement from both cohorts that asking a colleague for help was a mechanism by which interpersonal relationships were nurtured. In addition, there was general agreement among those who routinely used the KMS that the breadth of their knowledge in existing areas of knowledge and their knowledge in new areas were expanded as a consequence of KMS use. Those who did not routinely use the KMS agreed less frequently that KMS use broadened their breadth of technical knowledge.

\section{Conclusions}

Weak network ties were less prevalent among those who routinely used the KMS. A key difference between the physical characteristics of the networks of those who chose to use the KMS and those who did not was the level of network centrality. Behaviors indicative of information being concentrated with few individuals was lowest among non-routine KMS users $(\%$ of entropy $=3.5)$. Low measures of centrality are also indicative of weak ties. Granovetter claimed weak ties were a better source for innovative information than strong social ties [9]. The efficiency gained by having weak social ties enabled those who did not routinely use the KMS to achieve their goals without using the KMS. These behaviors may also reflect the longer tenure of respondents who indicated they did not routinely use the KMS. Having worked for the firm longer, respondents in this category developed information-seeking behaviors and interpersonal relationships which satisfied information requirements without the assistance of the KMS.

Information retrieval behaviors are shaped by geographic location. In addition, the legitimate role of potential information sources is a proxy for personal knowledge of others. Accordingly, respondents in medium and larger offices indicated they relied less on designations such as legitimate role in identifying potential information sources. In the absence of close physical proximity, the current and past role of an information source has the greatest impact on the information seeking behaviors where physical proximity constrains interaction.

Information seekers exclude potential information sources in efforts to improve information retrieval effectiveness. Contrary to social resource theory, few respondents sought information from their supervisors when solving an incident on behalf of their customers. In further contrast, actionable knowledge came from lower ranked individuals and was often obtained from organizationally-designated experts. Although a central premise of social resource theory was that those higher in the organizational hierarchy possessed better information [12], participants indicated they did not seek technical information from supervisors because they did not have current knowledge of the technologies supported by the firm. 
The use of IM complements both KMS use and colleague access. IM has been described as a "critical real-time communications tool" by which distance and time were overcome [13, p.1]. IM enables rapid dissemination of information, rapid problem resolution, immediate contact with experts, and is ideally suited for environments fraught with time sensitivity and geographic dispersal [7]. A greater proportion of routine KMS users participated in IM sessions (77\%) than those who did not routinely use the KMS (42\%). As a synchronous tool, IM expanded access to colleagues as potential information sources. At the same time, those who had a propensity to use the KMS, an asynchronous tool, also tended to leverage IM. New technologies do not necessarily result in the abandonment of previous ones [8]. Instead, IM services complemented the KMS.

There is evidence that synchronous and asynchronous technologies are converging, with long term implications for the traditional architecture of a KMS. Robot technologies are currently offered by AOL and AIM which automatically return responses based on messages sent from IM. The extent to which organizations are successful in integrating these new technologies into their processes become more important as the demographics of the workforce shift. The age of those who use technologies may significantly influence the features actually used [8]. While longer tenure did not necessarily mean those who did not routinely use the KMS were older than those who routinely used the KMS, the literature suggests this may have been the case.

Regardless of a respondent's propensity to use the KMS, a perceived cost of asking colleagues for help was an obligation to reciprocate. Consistent with social resource theory, reciprocity in the provision of information was universal among all respondents. The emotion of gratitude encouraged reciprocation "even if such reciprocation will be costly to him or her in the short term" [3, p. 319]. An interview participant described attempts to attain help from people she did not know as an effort to build and nurture relationships. Reciprocal prosocial behavior helps build trust and preserves relationships.

Perceptions of the technical prowess of colleagues are enhanced through KMS use. When the identity of the information provider is included in the KMS, the KMS may act as a proxy for direct interaction between an information seeker and the information provider. However, the mechanism by which a KMS mediates the human emotion of gratitude and impacts the construction of ongoing relationships requires further study.

At the individual level, balance between gaining information from a colleague verses a KMS is shaped by available technologies and the physical environment. One information source does not necessarily replace another, as information was sometimes gained through the complementary use of colleague referral and the KMS. Respondents preferred to learn from their peers. Nevertheless, the breadth of knowledge and knowledge in new areas was expanded through KMS use. Consistent with those of Zander and Kogut [17], the written and teachable nature of KMS content facilitated knowledge transfer. 


\section{Limitations}

The breadth of external validity of these conclusions needs to be established through further study. This study was conducted at multiple sites, within the same firm. It is unknown the extent to which conclusions drawn from the examination of one internal support organization can be generalized to other internal support organizations or in the open marketplace. Further consideration of organizational and geographic culture should be applied to related research in other contexts.

While efforts were made to encourage as much participation from potential participants as possible, ultimately responses to invitations for interviews or to complete surveys were voluntary. In addition, the author of this research conducted the interviews. Because he was associated with the KM tools being discussed, there is risk that the responses of respondents did not reflect their true feelings or perceptions.

\section{Recommendations for Practice and Further Research}

New tools do not necessarily replace old ones and the resultant levels of redundancy provide a mechanism by which all members of a community can participate in double-loop learning. Those responsible for encouraging collaboration within organizations should build redundancy into their knowledge sharing and learning platforms.

The acquisition of knowledge is not limited to the extraction of ideas from one source, such as a KMS, colleagues, or trial and error. Knowledge production can be facilitated through processes associated with social relationships, technologies, and direct experience. These processes may be organic or contrived in nature. Naturally occurring social networks or formalized knowledge refereeing and publication schemes are vehicles by which ideas are circulated within a work environment. Each methodology can contribute toward the integration of knowledge in the workplace. Those that influence or design such processes should be strategic when addressing the creation and propagation of these information paths.

Where great geographic disparity exists, information exchange is supported by relational structures that help people build and maintain ties along with corresponding mental directories. People in small offices recognized the expertise of others by virtue of formally designated roles; therefore, leaders in geographically dispersed organizations should consider purposeful and public designation of the expertise of members of the workforce when trying to build consistency across geographically-dispersed organizations.

Additional research is needed to understand the circumstances under which technologies can be leveraged to impact interpersonal network structure. Further research into the linkage between network centrality, social ties, and information 
exchange will provide guidance to practitioners seeking to improve the efficiency of information exchange across geographically-dispersed organizations. For both scholars and practitioners, the relevancy of further research in this area has expanded due to economic forces such as globalization, mergers, and acquisitions.

\section{References}

1. Alavi M, Kayworth T, Leidener D (2005) An empirical examination of the influence of organizational structure on knowledge management practices. J Management Inf Systems 22:191-224

2. Arnold V, Clark N, Collier P, Leech S, Sutton S (2006) The differential use and effect of knowledge-based system explanations in novice and expert judgment decisions. MIS Q 30:79-97

3. Bartlett M, DeSteno D (2006) Gratitude and proscocial behavior: Helping when it costs you. Psychological Science 17:319-325

4. Benta M (2005) Studying communication networks with Agna 2.1. Cognition, Brain, Behav 9:567-574

5. Borgatti S, Cross R (2003) A relational view of information seeking and learning in social networks. Management Science 49:432-445

6. Brown JS, Duguid P (1991) Organizational learning and communities-of-practice: Toward a unified view of working, learning, and innovation. Organizational Science 2:40-57

7. Cain M (2006) Justifying instant messaging investments. Technical Rep Gartner Res

8. Constantiou I, Damsgaard J, Knutsen L (2007) The four incremental steps toward advanced mobile service adoption. Communications of the ACM 50:51-55

9. Granovetter M (1973) The strength of weak ties. American J Sociology 78:1360-1380

10. Hargadon A, Sutton RI (1997) Technology brokering and innovation in a product development firm. Administrative Science Q 42:726-749

11. Keren G (1993) A balanced approach to unbalanced design. In: Keren G, Lewis C (eds) A handbook for data analysis in the behavior sciences. Lawrence Erlbaum Associates, Hillsdale, $\mathrm{NJ}$

12. Lin N (2001) Social capital: A theory of social structure and action. Cambridge University Press, New York

13. Lundy J, Smith DM (2007) The top five uses for instant messaging. Technical Rep Gartner Res

14. Michailova S, Husted K (2003) Knowledge-sharing hostility in Russian firms. Calif Management Rev 43:59-77

15. Prince S (2006) HP's adaptive approach to enterprise content management. KM World 388:12-13

16. Szulanski G, Cappetta R, Jensen R (2004) When and how trustworthiness matters: Knowledge transfer and the moderating effect of causal ambiguity. Organizational Science 15:600-613

17. Zander U, Kogut B (1995) Knowledge and the speed of transfer and imitation of organizational capabilities: An empirical test. Organizational Science 6:76-92 